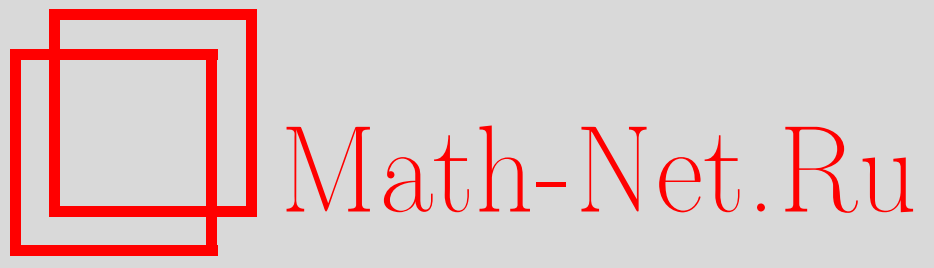

Г. К. Голубев, Ф. Н. Еникеева, Об одной задаче минимаксного оценивания дробной производной, Теория вероятн. и ее примен., 2001, том 46, выпуск 4, 658-677

DOI: https://doi.org/10.4213/tvp3793

Использование Общероссийского математического портала MathNet.Ru подразумевает, что вы прочитали и согласны с пользовательским соглашением

http: //www.mathnet.ru/rus/agreement

Параметры загрузки:

IP: 3.93 .64 .190

26 апреля 2023 г., 12:15:00

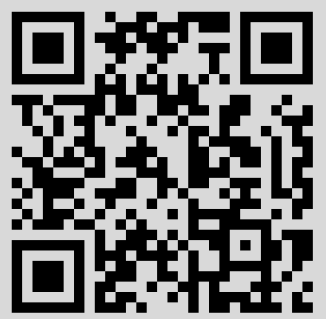


(C) $2001 \Gamma$.

ГОЛУБЕВ Г. К.*, ЕНИКЕЕВА Ф. Н. ${ }^{* *}$

\section{ОБ ОДНОЙ ЗАДАЧЕ МИНИМАКСНОГО ОЦЕНИВАНИЯ ДРОБНОЙ ПРОИЗВОДНОЙ}

Рассматривается задача минимаксного оценивания дробной производной порядка $-\frac{1}{2}$ неизвестной функции по наблюдениям в белом гауссовском шуме. Эта задача тесно связана с известной проблемой Виксела. В работе развивается асимптотически минимаксная теория второго порядка.

Ключевые слова и фразы: дробная производная, минимаксный риск второго порядка, задача Виксела.

\section{1. Введение}

В работе оценивается $f^{(\alpha)}(t)$ - дробная производная порядка $\alpha=-\frac{1}{2}$ неизвестной функции $f(t)$ по наблюдениям в белом гауссовском шуме

$$
d x(t)=f(t) d t+\varepsilon d w(t), \quad t \in[0,1], \quad x(0)=0 ;
$$

здесь $w(t)$ - стандартный винеровский процесс, а $\varepsilon$ - малый параметр. Наша задача - оценить $f^{(-1 / 2)}(t)$ при условии, что функция $f(t)$ принадлежит некоторому известному классу гладких функций. В действительности будут рассматриваться две задачи: оценивание $f^{(-1 / 2)}(t)$ в фиксированной точке $t_{0}$ и оценивание $f^{(-1 / 2)}(t)$ на всем отрезке $[0,1]$.

Чтобы упростить технические детали, предположим, что $f(t)-$ периодическая функция с нулевым средним, а $\varphi_{k}(t)=\exp (2 \pi i k t)-$ тригонометрический базис. Тогда, как хорошо известно [12], дробную производную порядка $\alpha$ можно определить следующим образом:

$$
f^{(\alpha)}(t)=\sum_{k=-\infty}^{\infty}\left\langle f, \varphi_{k}\right\rangle \varphi_{k}(t)(2 \pi i k)^{\alpha}
$$

${ }^{*}$ Институт проблем передачи информации РАН, Большой Каретный, 19, 101447 Москва, Россия; e-mail: glbv@iitp.ru

${ }^{* *}$ Московский государственный университет им. М.В. Ломоносова, механико-математический факультет, кафедра теории вероятностей, Воробьевы горы, 119899 Москва, Россия; e-mail: farida@shade.msu.ru 
где $\langle\cdot, \cdot\rangle$ - скалярное произведение в $\mathbf{L}_{2}(0,1)$. Пусть $\theta_{k}=\left\langle f, \varphi_{k}\right\rangle-$ коэффициенты Фурье функции $f$. Тогда задача оценивания производной порядка $-\frac{1}{2}$ в точке $t_{0}$ - это оценивание линейного функционала

$$
L(\theta)=\sum_{k=-\infty}^{\infty} \frac{\exp \left(2 \pi i k t_{0}\right)}{\sqrt{2 \pi i k}} \theta_{k}
$$

а задача оценивания производной того же порядка на всем отрезке сводится к задаче восстановления вектора $\left(\theta_{1} / \sqrt{1}, \theta_{2} / \sqrt{2}, \ldots\right)^{T}$.

$\mathrm{B}$ некоторых случаях задачу оценивания можно немного упростить, если перейти от наблюдений во временной области к наблюдениям в пространстве коэффициентов Фурье. Учитывая, что $\varphi_{k}(t)$ - полная ортонормированная система в. $\mathbf{L}_{2}(0,1)$, легко видеть, что наблюдения (1.1) эквивалентны следующим наблюдениям:

$$
X_{k}=\theta_{k}+\varepsilon \xi_{k}, \quad k=0, \pm 1, \pm 2, \ldots,
$$

где $\xi_{k}$ - независимые комплекснозначные гауссовские случайные величины с параметрами $(0,1)$,

$$
\xi_{k}=\int_{0}^{1} \varphi_{k}(t) d w(t), \quad X_{k}=\int_{0}^{1} \varphi_{k}(t) d x(t) .
$$

Важным ингредиентом любой статистической задачи является априорная информация о неизвестных параметрах. В настоящей работе предполагается, что функциональный класс, которому принадлежит неизвестная функция $f(t)$, - это эллипсоид в $\mathbf{L}_{2}(0,1)$. Точнее, считаем, что

$$
\theta \in \Theta=\left\{\theta: \sum_{k=1}^{\infty} a_{k}^{2}\left|\theta_{k}\right|^{2} \leqslant 1\right\} .
$$

В частности, если известно, что оцениваемая функция принадлежит соболевскому классу

$$
W_{2}^{\beta}=\left\{f: \int_{0}^{1}\left[f^{(\beta)}(t)\right]^{2} d t \leqslant P\right\}
$$

то в этом случае оси соответствуюшего эллипсоида определяются как $a_{k}^{2}=(2 \pi k)^{2 \beta} / P$.

На первый взгляд кажется, что рассматриваемая задача оценивания дробной производной поряцка - $\frac{1}{2}$ представляет довольно специальный интерес. В действительности она очень тесно связана с хорошо известной проблемой Виксела [10]. Последняя формулируется следующим образом. Пусть имеется непрозрачная среда, в которую помецены шары, радиусы которых являются независимыми одинаково распределенными случайными величинами с неизвестной функцией распределения $F(x)$. 
Интерес представляет именно $F(x)$. Однако, поскольку среда непрозрачна, предполагается, что мы не можем измерить непосредственно радиусы шаров. Можно только рассечь среду некоторой плоскостью и измерить радиусы шаров в сечении. Обозначим эти радиусы через $Y_{1}, \ldots, Y_{n}$. Задача - оценить по этим наблюдениям функцию распределения $F(x)$. Легко видеть, что величины $Y_{i}$ - независимые одинаково распределенные случайные величины с некоторой функцией распределения $G(y)$. Соотношения между $F$ и $G$ хорошо известны:

$$
\begin{aligned}
& 1-G(y)=\int_{y}^{\infty} \sqrt{x-y} d F(x)\left(\int_{0}^{\infty} \sqrt{x} d F(x)\right)^{-1}, \\
& 1-F(x)=\int_{y}^{\infty} \frac{d G(y)}{\sqrt{x-y}}\left(\int_{0}^{\infty} \frac{d G(y)}{\sqrt{y}}\right)^{-1} .
\end{aligned}
$$

С элементарным выводом этих формул можно познакомиться, например, в [3]. Эта формула на самом деле выражает неизвестную функцию распределения $F(x)$ через производные порядка $\frac{1}{2}$ от функции распределения $G(y)$ (присутствующие в ней интегралы - это просто другая эквивалентная форма записи дробных производных [12]). По сути дела, задача сводится к тому, чтобы по наблюдениям $Y_{1}, \ldots, Y_{n}$, имеющим неизвестную плотность $G(y)$, оценить $G^{(1 / 2)}(0)$ и $G^{(1 / 2)}(y)$. Эти величины являются, естественно, производными порядка $-\frac{1}{2}$ от плотности распределения $g(y)=G^{\prime}(y)$. Безусловно, эта задача не совпадает с рассматриваемой здесь задачей оценивания в белом шуме. Однако эти задачи очень близки. Хорошо известно, что соответствующие статистические эксперименты асимптотиंчески эквивалентны в смысле Ле Кама (см. [7]). Мы намеренно отбрасываем второстепенные технические детали и рассматриваем простейшую статистическую задачу, которая наиболее близка к проблеме Виксела, чтобы выяснить, как строить асимптотически минимаксные оценки второго порядка.

Заметим, что результаты об асимптотически минимаксных (при $n \rightarrow \infty$ ) оценках первого порядка в задаче Виксела были получены сравнительно недавно [3], хотя оптимальные скорости сходимости хорошо известны [6], [4], [2].

Цель настоящей работы - это построение асимптотически минимаксных оценок второго порядка в модели с белым гауссовским шумом. Перенесение результатов на задачу Виксела - дело хотя и не совсем тривиальное, но в основном техническое. Минимаксная теория второго порядка является в рассматриваемой задаче, по сушеству, довольно естественной. Дело в том, что существует очень много оценок, являющихся асимптотически минимаксными оценками первого порядка, и сказать, какая оценка является наилучшей в рамках теории первого порядка, невозможно. С другой стороны, асимптотически минимаксная оценка второго порядка уже является в некотором смысле единственной. 


\section{2. Постановка задачи и основные результаты}

Далее будем рассматривать несколько более общую постановку задачи, чем модель (1.2)-(1.3). Предположим, что наблюдаются действительные случайные величины

$$
X_{k}=\theta_{k}+\varepsilon \xi_{k}, \quad k=0,1, \ldots,
$$

где $\xi_{k}$ - гауссовские независимые случайные величины с параметрами $(0,1)$. Предполагается также, что неизвестный вектор $\theta=$ $\left(\theta_{1}, \theta_{2}, \ldots\right)^{T}$ принадлежит эллипсоиду

$$
\Theta=\left\{\theta: \sum_{k=1}^{\infty} a_{k}^{2} \theta_{k}^{2} \leqslant 1\right\},
$$

где величины $a_{k}^{2}$ считаются известными. С данной статистической моделью связаны две задачи.

Первая задача - поиск минимаксной оценки бесконечномерного вектора $v(\theta)=\left(\theta_{1} s_{1}, \theta_{2} s_{2}, \ldots\right)^{T}$, где последовательность $s_{k}$ такова, что

$$
\lim _{k \rightarrow \infty} s_{k}^{2} k=1 \text {. }
$$

Не оговаривая этого особо, считаем, что далее условие (2.3) выполнено. Обозначим через $\widehat{v}(X)=\left(\widehat{v}_{1}, \widehat{v}_{2}, \ldots\right)^{T}$ оценку вектора $v(\theta)$. Среднеквадратичный риск оценки $\widehat{v}$ определяется обычным образом:

$$
R^{\varepsilon}(\widehat{v}, \Theta)=\sup _{\theta \in \Theta} \mathbf{E}_{\theta}^{\varepsilon}\|v(\theta)-\widehat{v}(X)\|^{2}=\sup _{\theta \in \Theta} \mathbf{E}_{\theta}^{\varepsilon} \sum_{k=1}^{\infty}\left|v_{k}(\theta)-\widehat{v}_{k}\right|^{2},
$$

где $\mathbf{E}_{\theta}^{\varepsilon}-$ усреднение по мере, порожденной наблюдениями (2.1). Минимаксный риск в рассматриваемой задаче равен

$$
r^{\varepsilon}(\Theta)=\inf _{\hat{v}} R^{\varepsilon}(\widehat{v}, \Theta)
$$

где inf берется по всем оценкам вектора $v(\theta)$. Мы покажем, что при некоторых условиях линейные оценки являются асимптотически минимаксными оценками второго порядка, т.е.

$$
r^{\varepsilon}(\Theta)=\inf _{\hat{v} \in \mathscr{L}} R^{\varepsilon}(\widehat{v}, \Theta)+o\left(\varepsilon^{2}\right)
$$

здесь $\mathscr{L}$ - класс всех линейных оценок. Точнее, справедлив следующий результат.

Теорема 1. Пусть $\left|a_{k}\right|\left|s_{k}\right|$ не убывают $u$

$$
\begin{aligned}
& \lim _{\varepsilon \rightarrow 0} \ln ^{3} \frac{1}{\varepsilon^{2}} \sum_{k=1}^{\infty} a_{k}^{2}\left(\left|s_{k}\right|-\mu\left|a_{k}\right|\right)_{+}^{2}\left[\sum_{k=1}^{\infty}\left|a_{k}\right|\left(\left|s_{k}\right|-\mu\left|a_{k}\right|\right)_{+}\right]^{-2}=0 \\
& \lim _{\varepsilon \rightarrow 0} \frac{\max _{m}\left|a_{m}\right|\left(\left|s_{m}\right|-\mu\left|a_{m}\right|\right)_{+} \sum_{k=1}^{\infty}\left|a_{k}\right|\left(\left|s_{k}\right|-\mu\left|a_{k}\right|\right)_{+}}{\sum_{k=1}^{\infty} a_{k}^{2}\left(\left|s_{k}\right|-\mu\left|a_{k}\right|\right)_{+}^{2}}<\infty
\end{aligned}
$$




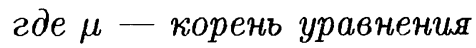

$$
\varepsilon^{2} \sum_{k=1}^{\infty} a_{k}^{2}\left(\frac{\left|s_{k}\right|}{\mu\left|a_{k}\right|}-1\right)_{+}=1
$$

Тогда линейная оченка

$$
\widetilde{v}_{k}=\left(1-\frac{\mu\left|a_{k}\right|}{\left|s_{k}\right|}\right)_{+} s_{k} X_{k}
$$

является асимптотически минимаксной оченкой второго порядка и

$$
r^{\varepsilon}(\Theta)=R^{\varepsilon}(\widetilde{v}, \Theta)+o\left(\varepsilon^{2}\right)=\varepsilon^{2} \sum_{k=1}^{\infty}\left|s_{k}\right|\left(\left|s_{k}\right|-\mu\left|a_{k}\right|\right)_{+}+o\left(\varepsilon^{2}\right)
$$

В частности, когда $a_{k}=(\pi k)^{\beta} / \sqrt{P}, \beta>\frac{1}{2}$, и $s_{k}=k^{-1 / 2}$, минимаксный риск имеет следующую асимптотику при $\varepsilon \rightarrow 0$ :

$$
r^{\varepsilon}(\Theta)=\frac{\varepsilon^{2}}{2 \beta+1} \ln \frac{(2 \beta+1) P}{\pi^{2 \beta} \varepsilon^{2}}+\varepsilon^{2}\left(\gamma-\frac{2}{2 \beta+1}\right)+o\left(\varepsilon^{2}\right)
$$

здесь и далее $\gamma$ - постоянная Эйлера.

Вторая задача связана с оцениванием линейного функционала

$$
L(\theta)=\sum_{k=1}^{\infty} \theta_{k} s_{k}
$$

Пусть $\widehat{L}(X)$ - некоторая оценка функционала $L$. Ее среднеквадратичный риск определяется как

$$
R_{0}^{\varepsilon}(\widehat{L}, \Theta)=\sup _{\theta \in \Theta} \mathbf{E}_{\theta}^{\varepsilon}[L(\theta)-\widehat{L}(X)]^{2}
$$

При этом минимаксный риск соответственно имеет вид

$$
r_{0}^{\varepsilon}(\Theta)=\inf _{\widehat{L}} R_{0}^{\varepsilon}(\widehat{L}, \Theta)
$$

где inf берется по всем оценкам функционала $L(\theta)$.

Следуюшая теорема дает верхнюю и нижнюю границы минимаксного риска.

Теорема 2. Минимаксный риск удовлетворяет следуюшим неравенствам:

$$
\varepsilon^{2} \sum_{k=1}^{\infty} s_{k}^{2}\left(1+\pi^{2} \varepsilon^{2} a_{k}^{2}\right)^{-1} \leqslant r_{0}^{\varepsilon}(\Theta) \leqslant \varepsilon^{2} \sum_{k=1}^{\infty} s_{k}^{2}\left(1+\varepsilon^{2} a_{k}^{2}\right)^{-1}
$$


Мы видим, что между верхней и нижней границей имеется зазор. Несложно проверить, что на соболевском шаре он равен $\left(\varepsilon^{2} \ln \pi\right) / \beta$. Наличие зазора связано с тем, что линейные оценки не являются минимаксными оценками второго порядка. К сожалению, явный вид минимаксной оценки в рассматриваемой задаче найти довольно трудно. Поэтому наш подход состоит в том, чтобы редуцировать исходную задачу к более простой статистической задаче. Такой задачей является оценивание функционала

$$
L_{\delta}(f)=\int_{\delta}^{\infty} \frac{f(t)}{\sqrt{t}} d t
$$

по наблюдениям в белом гауссовском шуме

$$
d X(t)=f(t) d t+d w(t), \quad t \in[0, \infty) .
$$

При этом априорная информация о $f(\cdot)$ имеет вид

$$
f \in \mathscr{F}=\left\{f \in \mathbf{L}_{2}(0, \infty): \int_{0}^{\infty} t^{2 \beta} f^{2}(t) d t \leqslant 1\right\} .
$$

Асимптотическое поведение минимаксного риска в исходной задаче с точностью до членов порядка $o\left(\varepsilon^{2}\right)$ описывается в терминах решения задачи об оценивании функционала $L_{\delta}(f)$ в задаче $(2.11)-(2.12)$. Обозначим через

$$
\rho=\lim _{\delta \rightarrow 0}\left\{\inf _{\widehat{L}_{\delta}} \sup _{f \in \mathscr{F}} \mathbf{E}_{\theta}\left[L_{\delta}(f)-\widehat{L}_{\delta}\right]^{2}+\ln \delta\right\}
$$

предельный минимаксный риск в задаче оценивания $L_{\delta}(f)$. Тогда справедлив следующий результат.

Теорема 3. Пусть $s_{k}=k^{-1 / 2} u a_{k}^{2}=(\pi k)^{2 \beta} P^{-1}(1+o(1)) n p u$ $k \rightarrow \infty$. Тогда при $\varepsilon \rightarrow 0$

$$
r_{0}^{\varepsilon}(\Theta)=\frac{\varepsilon^{2}}{2 \beta} \ln \frac{P}{\varepsilon^{2}}+\varepsilon^{2}(\gamma+\rho-\ln \pi)+o\left(\varepsilon^{2}\right) .
$$

\section{3. Оценивание производной на отрезке}

3.1. Верхняя граница. Доказательство теоремы 1 начнем с тривиальной верхней границы для минимаксного риска

$$
r^{\varepsilon}(\Theta) \leqslant \inf _{\widehat{v} \in \mathscr{L}} R^{\varepsilon}(\widehat{v}, \Theta) .
$$

Напомним, что здесь $\mathscr{L}-$ класс линейных оценок. Для того чтобы найти минимаксный риск в классе линейных оценок, воспользуемся хорошо известной теоремой о седловой точке [14]. В данном случае справедлива следующая лемма. 
Лемма 1. Оченка

$$
v_{k}^{*}=\left(1-\mu \frac{\left|a_{k}\right|}{\left|s_{k}\right|}\right)_{+} s_{k} X_{k},
$$

где $\mu$ - корень уравнения (2.7), является минимаксной в классе линейных оченок. При этом

$$
\inf _{\widehat{v} \in \mathscr{L}} R^{\varepsilon}(\widehat{v}, \Theta)=\varepsilon^{2} \sum_{k=1}^{\infty} s_{k}^{2}\left(1-\mu \frac{\left|a_{k}\right|}{\left|s_{k}\right|}\right)_{+} .
$$

Д о к а з а т е л ь с т в о. Среднеквадратичная ошибка линейной оценки $\widehat{v}_{k}=h_{k} s_{k} X_{k}$

$$
\mathbf{E}_{\theta} \sum_{k=1}^{\infty}\left|v_{k}-\widehat{v}_{k}\right|^{2}=\sum_{k=1}^{\infty} s_{k}^{2}\left(1-h_{k}\right)^{2} \theta_{k}^{2}+\varepsilon^{2} \sum_{k=1}^{\infty} h_{k}^{2} s_{k}^{2}=F^{\varepsilon}(h, \theta)
$$

представляет собой функционал, выпуклый по $h$ и линейный по $\theta_{k}^{2}$. Следовательно, он имеет седловую точку на множестве $l_{2}(1, \infty) \times \Theta$. Простые арифметические выкладки (см., например, [14]), которые мы опустим, показывают, что компоненты седловой точки вычисляются следующим образом:

$$
h_{k}^{*}=\left(1-\mu \frac{\left|a_{k}\right|}{\left|s_{k}\right|}\right)_{+}, \quad \theta_{k}^{* 2}=\frac{\varepsilon^{2} h_{k}^{*}}{1-h_{k}^{*}}=\varepsilon^{2}\left(\frac{\left|s_{k}\right|}{\dot{\mu}\left|a_{k}\right|}-1\right)_{+} ;
$$

здесь $\mu$, естественно, корень уравнения $\sum_{k=1}^{\infty} a_{k}^{2} \theta_{k}^{* 2}=1$. Поэтому, замечая, что

$$
\inf _{\widehat{v} \in \mathscr{L}} R^{\varepsilon}(\widehat{v}, \Theta)=\inf _{h} \sup _{\theta \in \Theta} F^{\varepsilon}(h, \theta)=F^{\varepsilon}\left(h^{*}, \theta^{*}\right)=\varepsilon^{2} \sum_{k=1}^{\infty} s_{k}^{2} h_{k}^{*},
$$

мы завершаем доказательство леммы.

3.2. Нижняя граница. Теперь установим нижнюю границу минимаксного риска. При этом мы будем следовать [14]. Выберем априорное распределение параметров $\theta_{k}$ так, чтобы дисперсия $\theta_{k}$ была близка к седловой точке (3.2) и чтобы вектор $\theta$ лежал вблизи поверхности эллипсоида (2.2). Точнее, предположим, что $\theta_{k}$ имеют нормальное распределение с параметрами $\left(0, \sigma_{k}^{2}\right)$, где

$$
\sigma_{k}^{2}=(1-\delta) \varepsilon^{2}\left(\frac{\left|s_{k}\right|}{\mu\left|a_{k}\right|}-1\right)_{+}, \quad 0<\delta<1
$$

а $\mu-$ корень уравнения (2.7). Заметим, что при $\delta=0$ дисперсия $\theta_{k}$ совпадает с седловой точкой (3.2), которая определяет минимаксіную линейную оценку. Прежде всего, покажем, что при малых $\delta>0$ вероятность того, что вектор $\theta$ не принадлежит эллипсоиду, стремится к 0 квалифицированным образом при $\varepsilon \rightarrow 0$. 
Лемма 2. Пусть $\left|a_{k} s_{k}\right|$ не убывают и выполнено условие (2.6). Тогда для любого $\delta \in\left(0, \delta_{0}\right)$

$$
\mathbf{P}\{\theta \notin \Theta\} \leqslant \exp \left(-\frac{\delta^{2}}{4 w_{\varepsilon}}\right), \quad \text { əдe } w_{\varepsilon}=\sum_{k=1}^{\infty} a_{k}^{4} \sigma_{k}^{4}
$$

Д ок а з а тель с т о. Заметим, что

$$
\mathbf{P}\{\theta \notin \Theta\}=\mathbf{P}\left\{\sum_{k=1}^{\infty} a_{k}^{2} \theta_{k}^{2}>1\right\}=\mathbf{P}\left\{\sum_{k=1}^{\infty} a_{k}^{2}\left(\theta_{k}^{2}-\sigma_{k}^{2}\right)>\delta\right\} .
$$

Согласно неравенству Чебышева, имеем, что при всех $\lambda$ таких, что $2 \lambda \sup _{k} a_{k}^{2} \sigma_{k}^{2}<1$

$$
\begin{aligned}
\mathbf{P}\{\theta \notin \Theta\} & \leqslant e^{-\lambda \delta} \mathbf{E} \exp \left[\lambda \sum_{k=1}^{\infty} a_{k}^{2}\left(\theta_{k}^{2}-\sigma_{k}^{2}\right)\right] \\
& =e^{-\lambda \delta} \exp \left[-\lambda \sum_{k=1}^{\infty} a_{k}^{2} \sigma_{k}^{2}-\frac{1}{2} \sum_{k=1}^{\infty} \ln \left(1-2 \lambda a_{k}^{2} \sigma_{k}^{2}\right)\right] .
\end{aligned}
$$

Выберем $\lambda=\delta /\left(2 \sum_{k=1}^{\infty} a_{k}^{4} \sigma_{k}^{4}\right)$ и проверим, что $1-2 \lambda a_{k}^{2} \sigma_{k}^{2}>0$ при достаточно малых $\delta$. Для этого достаточно, чтобы было выполнено неравенство

$$
\sup _{k} a_{k}^{2} \sigma_{k}^{2}\left(\sum_{k=1}^{\infty} a_{k}^{4} \sigma_{k}^{4}\right)^{-1}<\infty .
$$

Из (3.3) и (2.7) получаем, что

$$
\frac{\max _{k} a_{k}^{2} \sigma_{k}^{2}}{\sum_{k=1}^{\infty} a_{k}^{4} \sigma_{k}^{4}}=\frac{\max _{m}\left|a_{m}\right|\left(\left|s_{m}\right|-\mu\left|a_{m}\right|\right)_{+} \cdot \sum_{k=1}^{\infty}\left|a_{k}\right|\left(\left|s_{k}\right|-\mu\left|a_{k}\right|\right)_{+}}{(1-\delta) \sum_{k=1}^{\infty}\left|a_{k}\right|^{2}\left(\left|s_{k}\right|-\mu\left|a_{k}\right|\right)_{+}^{2}}
$$

В силу условия (2.6) правая часть в этом равенстве ограничена, и поэтому выполнено (3.6). Тогда из (3.5) и разложения Тейлора получаем

$$
\mathbf{P}\{\theta \notin \Theta\} \leqslant e^{-\lambda \delta} \exp \left(\lambda^{2} \sum_{k=1}^{\infty} a_{k}^{4} \sigma_{k}^{4}\right)=\exp \left\{-\frac{\delta^{2}}{4 \sum_{k=1}^{\infty} a_{k}^{4} \sigma_{k}^{4}}\right\},
$$

тем самым доказывая лемму.

Лемма 3. Пусть выполнены условия (2.5) $u(2.6)$, тогда при $\varepsilon \rightarrow 0$

$$
r^{\varepsilon}(\Theta) \geqslant \varepsilon^{2} \sum_{k=1}^{\infty} s_{k}^{2}\left(1-\mu \frac{\left|a_{k}\right|}{\left|s_{k}\right|}\right)_{+}+o\left(\varepsilon^{2}\right)
$$

где $\mu-$ корень уравнения (2.7). 
Д ок а з а т е ль с т в о. Пусть $\hat{\theta}_{k}$ - оценка параметра $\theta_{k}$. Тогда, согласно неравенству треугольника, минимаксный риск оценивается снизу следуюшим образом:

$$
r^{\varepsilon}(\Theta)=\inf _{\hat{\theta}} \sup _{\theta \in \Theta} \mathbf{E}_{\theta} \sum_{k=1}^{\infty} s_{k}^{2}\left(\theta_{k}-\hat{\theta}_{k}\right)^{2} \geqslant \inf _{\hat{\theta} \in \Theta} \sup _{\theta \in \Theta} \mathbf{E}_{\theta} \sum_{k=1}^{\infty} s_{k}^{2}\left(\theta_{k}-\hat{\theta}_{k}\right)^{2} .
$$

Так как

$$
\sup _{\theta \in \Theta} \mathbf{E}_{\theta} \sum_{k=1}^{\infty} s_{k}^{2}\left(\theta_{k}-\hat{\theta}_{k}\right)^{2} \geqslant \mathbf{E} \mathbf{E}_{\theta} \mathbf{1}\{\theta \in \Theta\} \sum_{k=1}^{\infty} s_{k}^{2}\left(\theta_{k}-\hat{\theta}_{k}\right)^{2},
$$

то неравенство (3.8) можно продолжить следующим образом:

$$
\begin{aligned}
r^{\varepsilon}(\Theta) \geqslant & \inf _{\hat{\theta} \in \Theta} \mathbf{E} \mathbf{E}_{\theta} \mathbf{1}\{\theta \in \Theta\} \sum_{k=1}^{\infty} s_{k}^{2}\left(\theta_{k}-\hat{\theta}_{k}\right)^{2} \geqslant \inf _{\hat{\theta} \in \Theta} \mathbf{E} \mathbf{E}_{\theta} \sum_{k=1}^{\infty} s_{k}^{2}\left(\theta_{k}-\hat{\theta}_{k}\right)^{2} \\
& -\sup _{\hat{\theta} \in \Theta} \mathbf{E} \mathbf{E}_{\theta} \mathbf{1}\{\theta \notin \Theta\} \sum_{k=1}^{\infty} s_{k}^{2}\left(\theta_{k}-\hat{\theta}_{k}\right)^{2} .
\end{aligned}
$$

Так как $\theta_{k}$ - независимые $\mathscr{N}\left(0, \sigma_{k}^{2}\right)$ с $\sigma_{k}^{2}$ из (3.3), то $\theta_{k}=\sqrt{1-\delta} \theta_{k}^{*} \xi_{k}$, где $\xi_{k}$ - независимые $\mathscr{N}(0,1)$, а $\theta_{k}^{*}$ - седловая точка (см. (3.2)). Отсюда, воспользовавшись леммой 1 , мы получаем

$$
\begin{aligned}
\inf _{\hat{\theta} \in \Theta} \mathbf{E} \mathbf{E}_{\theta} \sum_{k=1}^{\infty} s_{k}^{2}\left(\theta_{k}-\hat{\theta}_{k}\right)^{2} & \geqslant \inf _{\hat{\theta}} \mathbf{E} \mathbf{E}_{\theta} \sum_{k=1}^{\infty} s_{k}^{2}\left(\sqrt{1-\delta} \theta_{k}^{*} \xi_{k}-\hat{\theta}_{k}\right)^{2} \\
& \geqslant(1-\delta) \inf _{\hat{\theta}} \mathbf{E} \mathbf{E}_{\theta} \sum_{k=1}^{\infty} s_{k}^{2}\left(\theta_{k}^{*} \xi_{k}-\hat{\theta}_{k}\right)^{2} \\
& =(1-\delta) \inf _{h_{k}} \mathbf{E} \mathbf{E}_{\theta} \sum_{k=1}^{\infty} s_{k}^{2}\left(\theta_{k}^{*} \xi_{k}-h_{k} X_{k}\right)^{2} \\
& =(1-\delta) \varepsilon^{2} \sum_{k=1}^{\infty} s_{k}^{2}\left(1-\mu \frac{\left|a_{k}\right|}{\left|s_{k}\right|}\right)_{+}
\end{aligned}
$$

Чтобы оценить снизу последнее слагаемое в правой части (3.9), заметим, что поскольку $\theta_{k}$ - гауссовские случайные величины, мы имеем

$$
\mathbf{E}\left(\sum_{k=1}^{\infty} s_{k}^{2} \theta_{k}^{2}\right)^{2}=3 \sum_{k=1}^{\infty} s_{k}^{4} \sigma_{k}^{4}+2 \sum_{k \neq l} s_{k}^{2} s_{l}^{2} \sigma_{k}^{2} \sigma_{l}^{2} \leqslant 3\left(\sum_{k=1}^{\infty} s_{k}^{2} \sigma_{k}^{2}\right)^{2} \leqslant C .
$$

Из этого неравенства и из неравенства Коши-Буняковского находим

$$
\begin{aligned}
& \sup _{\hat{\theta} \in \Theta} \mathbf{E} \mathbf{E}_{\theta} \mathbf{1}\{\theta \notin \Theta\} \sum_{k=1}^{\infty} s_{k}^{2}\left(\theta_{k}-\hat{\theta}_{k}\right)^{2} \\
& \quad \leqslant[\mathbf{P}\{\theta \notin \Theta\}]^{1 / 2} \sup _{\hat{\theta} \in \Theta}\left\{\mathbf{E E}_{\theta}\left[\sum_{k=1}^{\infty} s_{k}^{2}\left(\theta_{k}-\hat{\theta}_{k}\right)^{2}\right]^{2}\right\}^{1 / 2} \leqslant C[\mathbf{P}\{\theta \notin \Theta\}]^{1 / 2} .
\end{aligned}
$$


Поэтому отсюда, из (3.9), (3.10) и леммы 2 вытекает, что для некоторой постоянной $C$

$$
r^{\varepsilon}(\Theta) \geqslant(1-\delta) \varepsilon^{2} \sum_{k=1}^{\infty} s_{k}^{2}\left(1-\mu \sqrt{k}\left|a_{k}\right|\right)_{+}-C \exp \left(-\frac{\delta^{2}}{C w_{\varepsilon}}\right) .
$$

«Поднимем» нижнюю границу, максимизируя правую часть этого неравенства по $\delta$. Обозначим для краткости

$$
\rho^{\varepsilon}=\varepsilon^{2} \sum_{k=1}^{\infty} s_{k}^{2}\left(1-\mu \sqrt{k}\left|a_{k}\right|\right)_{+}
$$

тогда, выбрав $\delta=\sqrt{-C_{0} w_{\varepsilon} \ln \left(\rho^{\varepsilon} w_{\varepsilon}\right)}$, где $C_{0}$ - достаточно большое число, несложно проверить, что

$$
\begin{aligned}
& \min _{\delta}\left\{\delta \rho^{\varepsilon}+C \exp \left(-\frac{\delta^{2}}{C w_{\varepsilon}}\right)\right\} \leqslant \rho^{\varepsilon} \sqrt{w_{\varepsilon}} \ln ^{1 / 2} \frac{1}{\rho^{\varepsilon} w_{\varepsilon}} \\
& \leqslant C \rho^{\varepsilon} \sqrt{w_{\varepsilon}} \ln ^{1 / 2} \frac{1}{\rho^{\varepsilon} \sqrt{w_{\varepsilon}}} .
\end{aligned}
$$

С другой стороны, согласно (2.7), находим

$$
\begin{aligned}
w_{\varepsilon} & =\sum_{k=1}^{\infty} a_{k}^{4} \sigma_{k}^{4}=\frac{\varepsilon^{2}}{\mu^{2}} \sum_{k=1}^{\infty} a_{k}^{2} s_{k}^{2}\left(1-\mu \frac{\left|a_{k}\right|}{\left|s_{k}\right|}\right)_{+}^{2} \\
& =\sum_{k=1}^{\infty} a_{k}^{2} s_{k}^{2}\left(1-\mu \frac{\left|a_{k}\right|}{\left|s_{k}\right|}\right)_{+}^{2}\left[\sum_{k=1}^{\infty}\left|a_{k}\right|\left|s_{k}\right|\left(1-\mu \frac{\left|a_{k}\right|}{\left|s_{k}\right|}\right)_{+}\right]^{-2} .
\end{aligned}
$$

Чтобы продолжить неравенство (3.12), определим целое число

$$
N=\min \left\{k: 1-\mu \frac{\left|a_{k}\right|}{\left|s_{k}\right|}<0\right\} \text {. }
$$

Очевидно, что $\rho^{\varepsilon} \leqslant \varepsilon^{2} \ln N$, и из уравнения (2.7) получаем, что $N<\varepsilon^{-2}$. Поэтому $\rho^{\varepsilon} \leqslant \varepsilon^{2} \ln \varepsilon^{-2}$. Отсюда, из $(3.11),(3.12)$ и (2.5) получаем требуемое неравенство (3.7).

Д о к а за т е л с т в о т е о р е м ы 1 вытекает непосредственно из лемм 1 и 3.

3.3. Асимптотика на соболевском классе. В этом пункте мы более детально рассмотрим асимптотику минимаксного риска на соболевском классе, считая, что $a_{k}=(\pi k)^{\beta} / \sqrt{P}$, где $\beta>\frac{1}{2}$, а $s_{k}=k^{-1 / 2}$.

Пусть величина $N$ определена.в (3.14). Тогда $N$ и $\mu$ связаны простым соотношением $\mu=(1+o(1))\left|s_{N}\right| /\left|a_{N}\right|$ при $\varepsilon \rightarrow 0$. Поэтому уравнение (2.7) для $N$ принимает вид

$$
\varepsilon^{2} \sum_{k=1}^{N} a_{k}^{2}\left(\frac{\left|s_{k} a_{N}\right|}{\left|a_{k} s_{N}\right|}-1\right)=1
$$


Отсюда мы получаем следующее уравнение для $N$ :

$$
\sum_{k=1}^{N} k^{2 \beta}\left[\left(\frac{N}{k}\right)^{\beta+1 / 2}-1\right]=\frac{P}{\pi^{2 \beta} \varepsilon^{2}} .
$$

С помощью простой арифметики нетрудно убедиться, что при $\varepsilon \rightarrow 0$

$$
N=(1+o(1))\left[\frac{P(2 \beta+1)}{\pi^{2 \beta} \varepsilon^{2}}\right]^{1 /(2 \beta+1)} .
$$

Теперь условия (2.5) и (2.6) легко проверяются, если заметить, что

$$
\begin{aligned}
\sum_{k=1}^{\infty}\left|a_{k} s_{k}\right|\left(1-\mu \frac{\left|a_{k}\right|}{\left|s_{k}\right|}\right)_{+} & \asymp \sum_{k=1}^{N} k^{\beta-1 / 2}\left[1-\left(\frac{k}{N}\right)^{\beta+1 / 2}\right] \asymp N^{\beta+6 / 2} \\
\sum_{k=1}^{\infty} a_{k}^{2} s_{k}^{2}\left(1-\mu \frac{\left|a_{k}\right|}{\left|s_{k}\right|}\right)_{+}^{2} & \asymp \sum_{k=1}^{N} k^{2 \beta-1}\left[1-\left(\frac{k}{N}\right)^{\beta+1 / 2}\right]^{2} \asymp N^{2 \beta} \\
\max _{k}\left|a_{k} s_{k}\right|\left(1-\mu \frac{\left|a_{k}\right|}{\left|s_{k}\right|}\right)_{+} & \asymp \max _{k} k^{\beta-1 / 2}\left[1-\left(\frac{k}{N}\right)^{\beta+1 / 2}\right] \asymp N^{\beta-1 / 2} .
\end{aligned}
$$

Изучим асимптотическое поведение минимаксного риска при $\varepsilon \rightarrow 0$. Для любого числа $\delta \in(0,1)$ имеем

$$
\begin{aligned}
r^{\varepsilon}(\Theta) & =\varepsilon^{2} \sum_{k=1}^{N} s_{k}^{2}\left(1-\mu \frac{\pi^{\beta} k^{\beta}}{\left|s_{k}\right| \sqrt{P}}\right)=\varepsilon^{2} \sum_{k=1}^{N} s_{k}^{2}\left[1-\left(\frac{k}{N}\right)^{\beta+1 / 2}\right]+o\left(\varepsilon^{2}\right) \\
& =\varepsilon^{2} \sum_{k=1}^{\delta N} s_{k}^{2}\left[1-\left(\frac{k}{N}\right)^{\beta+1 / 2}\right]+\varepsilon^{2} \sum_{k=\delta N+1}^{N} s_{k}^{2}\left[1-\left(\frac{k}{N}\right)^{\beta+1 / 2}\right]+o\left(\varepsilon^{2}\right) \\
& \equiv \varepsilon^{2}\left[r_{1}(\varepsilon, \delta)+r_{2}(\varepsilon, \delta)\right]+o\left(\varepsilon^{2}\right) .
\end{aligned}
$$

Оценим $r_{1}(\varepsilon, \delta)$. Поскольку $k \leqslant \delta N$, то $1-(k / N)^{\beta+1 / 2}=1+O\left(\delta^{\beta+1 / 2}\right)$ при $\delta \rightarrow 0$. Следовательно,

$$
r_{1}(\varepsilon, \delta)=\ln N+\gamma+\ln \delta+O\left(\delta^{\beta+1 / 2} \ln N\right)+o(1) .
$$

Рассмотрим теперь $r_{2}(\varepsilon, \delta)$. Легко видеть, что

$$
\begin{aligned}
r_{2}(\varepsilon, \delta) & =\int_{\delta}^{1} x^{-1}\left(1-x^{\beta+1 / 2}\right) d x+O\left(N^{-1} \delta^{-1}\right) \\
& =-\ln \delta-\frac{2}{2 \beta+1}\left(1-\delta^{\beta+1 / 2}\right)+O\left(N^{-1} \delta^{-1}\right) .
\end{aligned}
$$

Выберем $\delta=(\ln N)^{-1-q}$, где $q>0$. Тогда из (3.16) и (3.17) находим, что

$$
r_{1}(\varepsilon, \delta)+r_{2}(\varepsilon, \delta)=\ln N+\gamma-\frac{2}{2 \beta+1}+o(1) .
$$


Поэтому, учитывая (3.15), получаем, что при $\varepsilon \rightarrow 0$ справедливо следующее разложение для минимаксного риска:

$$
r^{\varepsilon}(\Theta)=\frac{\varepsilon^{2}}{2 \beta+1} \ln \frac{P(2 \beta+1)}{\varepsilon^{2} \pi^{2 \beta}}+\varepsilon^{2}\left(\gamma-\frac{2}{2 \beta+1}\right)+o\left(\varepsilon^{2}\right) .
$$

\section{4. Оценивание производной в точке}

4.1. Верхняя граница. Верхнюю границу для минимаксного риска $r_{0}^{\varepsilon}(\Theta)$ в задаче оценивания линейного функционала $L(\theta)=$ $\sum_{k=1}^{\infty} s_{k} \theta_{k}$ будем искать в классе линейных оценок $\mathscr{L}$.

Лемма 4. Минимаксный риск оченивания функиионала $L(\theta)$ в классе линейных оченок равен

$$
\inf _{\widehat{L} \in \mathscr{L}} R_{0}^{\varepsilon}(\widehat{L}, \Theta)=\varepsilon^{2} \sum_{k=1}^{\infty} s_{k}^{2}\left(1+\varepsilon^{2} a_{k}^{2}\right)^{-1}
$$

При этом оченка

$$
\widehat{L}_{h}(X)=\sum_{k=1}^{\infty}\left(1+\varepsilon^{2} a_{k}^{2}\right)^{-1} s_{k} X_{k}
$$

является минимаксной линейной оченкой.

Д ок азат е льс т в о. Рассмотрим $\widehat{L}_{h}(X)=\sum_{k=1}^{\infty} h_{k} s_{k} X_{k}$. Легко видеть, что

$$
R_{0}^{\varepsilon}\left(\widehat{L}_{h}, \Theta\right)=\sup _{\theta \in \Theta}\left[\sum_{k=1}^{\infty} \theta_{k}\left(1-h_{k}\right) s_{k}\right]^{2}+\varepsilon^{2} \sum_{k=1}^{\infty} h_{k}^{2} s_{k}^{2} .
$$

Отсюда, пользуясь неравенством Коши-Буняковского, получаем, что

$$
\begin{aligned}
R_{0}^{\varepsilon}\left(\widehat{L}_{h}, \Theta\right) & =\sum_{k=1}^{\infty} a_{k}^{2} \theta_{k}^{2} \sum_{k=1}^{\infty} a_{k}^{-2}\left(1-h_{k}\right)^{2} s_{k}^{2}+\varepsilon^{2} \sum_{k=1}^{\infty} h_{k}^{2} s_{k}^{2} \\
& =\sum_{k=1}^{\infty} a_{k}^{-2} s_{k}^{2}\left(1-h_{k}\right)^{2}+\varepsilon^{2} \sum_{k=1}^{\infty} h_{k}^{2} s_{k}^{2}
\end{aligned}
$$

Легко видеть, что минимум по $h_{k}$ правой части достигается при $\widehat{h}_{k}=$ $\left(1+\varepsilon^{2} a_{k}^{2}\right)^{-1}$. Отсюда вытекает (4.1).

4.2. Нижняя граница. Найдем теперь нижнюю границу для минимаксного риска $r_{0}^{\varepsilon}(\Theta)$. При этом мы используем стандартный подход [13]. Будем считать, что $\theta_{k}=\theta b_{k}$, где $\theta-$ некоторая случайная величина, $b_{k}$ - некоторая фиксированная последовательность. Тогда по наблюдениям $X_{k}=\theta b_{k}+\varepsilon \xi_{k}$ нужно оценить параметр

$$
L(\theta)=\theta \sum_{k=1}^{\infty} b_{k} s_{k}
$$


Заметим, что $\sum_{k=1}^{\infty} b_{k} X_{k}$ - достаточная статистика, и поэтому можно оценивать параметр $\theta$ всего лишь по одному наблюдению

$$
Y=\sum_{k=1}^{\infty} b_{k} X_{k}=\theta \sum_{k=1}^{\infty} b_{k}^{2}+\varepsilon^{2} \sum_{k=1}^{\infty} b_{k} \xi_{k}
$$

Отсюда получаем эквивалентную задачу оценки параметра $L(\theta)$ (см. (4.2)) по наблюдению

$$
Y^{\prime}=\theta+\varepsilon^{2} \xi\|b\|^{-1}
$$

где $\xi \sim \mathscr{N}(0,1)$ и $\|\cdot\|$ - норма в $l_{2}(1, \infty)$. При этом условие $(2.2)$ влечет следующее ограничение на $\theta$ :

$$
\theta^{2} \leqslant\left(\sum_{k=1}^{\infty} a_{k}^{2} b_{k}^{2}\right)^{-1}
$$

Лемма 5. Нижняя граница для минимаксного риска в задаче оченивания функиионала $L(\theta)$ имеет вид

$$
r_{0}^{\varepsilon}(\Theta) \geqslant \varepsilon^{2} \sum_{k=1}^{\infty} s_{k}^{2}\left(1+\varepsilon^{2} \pi^{2} a_{k}^{2}\right)^{-1}
$$

Д о к а $з$ а те ль с т в о. Обозначим $A=\left(\sum_{k=1}^{\infty} a_{k}^{2} b_{k}^{2}\right)^{-1 / 2}$. Заметим, что

$$
\begin{aligned}
r_{0}^{\varepsilon}(\Theta) & \geqslant \inf _{\widehat{L}} \sup _{|\theta| \leqslant A} \mathbf{E}_{\theta}\left[\theta \sum_{k=1}^{\infty} b_{k} s_{k}-\widehat{L}\left(Y^{\prime}\right)\right]^{2} \\
& =\left(\sum_{k=1}^{\infty} b_{k} s_{k}\right)^{2} \text { inf } \sup _{\hat{\theta}} \mathbf{E}_{\theta}(\theta-\hat{\theta})^{2}
\end{aligned}
$$

Пусть $\nu$ - некоторая априорная плотность распределения параметра $\theta$ такая, что носитель $\nu$ принадлежит $[-A, A]$, тогда

$$
\sup _{|\theta| \leqslant A} \mathbf{E}_{\theta}(\theta-\hat{\theta})^{2} \geqslant \int \mathbf{E}_{\theta}(\theta-\hat{\theta})^{2} \nu(\theta) d \theta .
$$

Далее, из неравенства Ван Триса [11] получаем

$$
\int \mathbf{E}_{\theta}(\theta-\hat{\theta})^{2} \nu(\theta) d \theta \geqslant\left[\mathbf{E} I\left(p_{\theta}\right)+I(\nu)\right]^{-1},
$$

где фишеровские информации в правой части этого неравенства соответственно равны

$$
I\left(p_{\theta}\right)=\int \frac{{p_{\theta}^{\prime}}^{2}(x)}{p_{\theta}(x)} d x, \quad I(\nu)=\int_{-A}^{A} \frac{\nu^{\prime 2}(x)}{\nu(x)} d x
$$


здесь $p_{\theta}(\cdot)$ - плотность распределения наблюдений из (4.3). Минимизируя фишеровскую информацию $I(\nu)$ по априорной плотности $\nu$, нетрудно видеть, что $\inf _{\nu} I(\nu)=I\left(\nu^{*}\right)=\pi^{2} A^{2}$, где $\nu^{*}(x)=A^{-1} \cos ^{2}[\pi x /(2 A)]$. Так как $\mathbf{E} I\left(p_{\theta}\right)=\|b\|^{2} / \varepsilon^{2}$, то из (4.6)-(4.8), получаем, что

$$
r_{0}^{\varepsilon}(\Theta) \geqslant\left(\sum_{k=1}^{\infty} b_{k} s_{k}\right)^{2}\left(\|b\|^{2} \varepsilon^{-2}+\pi^{2} \sum_{k=1}^{\infty} a_{k}^{2} b_{k}^{2}\right)^{-1} .
$$

«Поднимем» эту нижнюю границу, максимизировав правую часть неравенства по $b_{\kappa}$. Легко видеть, что максимум достигается при $b_{k}^{*}=$ $s_{k}\left(\varepsilon^{-2}+\pi^{2} a_{k}^{2}\right)^{-1}$. Отсюда получаем (4.5).

Д ок а з а те ль с т в о т е о ре мы 2 очевидным образом следует из лемм 4 и 5.

4.3. Асимптотика на соболевском классе. Исследуем поведение полученных границ для минимаксного риска при $\varepsilon \rightarrow 0$, когда $a_{k}=(\pi k)^{\beta} / \sqrt{P}$ и $s_{k}=k^{-1 / 2}$. Пусть $N=\max \left\{k:\left|a_{k}\right|<1 / \varepsilon\right\}$, тогда мы имеем

$$
\begin{aligned}
\sum_{k=1}^{\infty} s_{k}^{2}\left(1+\varepsilon^{2} a_{k}^{2}\right)^{-1} & =\varepsilon^{2} \sum_{k=1}^{\delta N} k^{-1}\left(1+\varepsilon^{2} a_{k}^{2}\right)^{-1}+\varepsilon^{2} \sum_{k=\delta N+1}^{\infty} k^{-1}\left(1+\varepsilon^{2} a_{k}^{2}\right)^{-1} \\
& \equiv \varepsilon^{2}\left[S_{1}(\varepsilon, \delta)+S_{2}(\varepsilon, \delta)\right]
\end{aligned}
$$

где $\delta \in(0,1)$ - некоторое число, зависящее от $\varepsilon$, которое выберем позднее. Заметим, что при $\varepsilon \rightarrow 0$

$$
S_{1}(\varepsilon, \delta)=\sum_{k=1}^{\delta N} k^{-1}+O\left(\delta^{2 \beta}\right)=\ln N+\ln \delta+\gamma+O\left(\delta^{2 \beta}\right) .
$$

Учитывая, что $N=(1+o(1)) \pi^{-1}\left(P / \varepsilon^{2}\right)^{1 /(2 \beta)}$, получаем

$$
\begin{aligned}
S_{2}(\varepsilon, \delta) & =\sum_{k=\delta N+1}^{\infty} k^{-1}\left[1+\frac{\varepsilon^{2}(\pi k)^{2 \beta}}{P}\right]^{-1} \\
& =\int_{\delta}^{\infty} x^{-1}\left(1+x^{2 \beta}\right)^{-1} d x+O\left(N^{-1} \delta^{-1}\right) .
\end{aligned}
$$

С другой стороны, легко показать, что

$$
\int_{\delta}^{\infty} x^{-1}\left(1+x^{2 \beta}\right)^{-1} d x=-\ln \delta+(2 \beta)^{-1} \ln \left(1+\delta^{2 \beta}\right) .
$$

Поэтому, выбрав, например, $\delta=\ln ^{-1} \varepsilon^{-2}$, находим из (4.9)-(4.11) асимптотику верхней границы

$$
r_{0}^{\varepsilon}(\Theta) \leqslant \sum_{k=1}^{\infty} s_{k}^{2}\left(1+\varepsilon^{2} a_{k}^{2}\right)^{-1}=\frac{\varepsilon^{2}}{2 \beta} \ln \frac{P}{\varepsilon^{2}}+\varepsilon^{2}(\gamma-\ln \pi)+o\left(\varepsilon^{2}\right) .
$$


Очевидно, что для нижней границы (4.5) асимптотика будет иметь аналогичное разложение, только $P$ в (4.12) надо заменить на $P / \pi^{2}$. То есть при $\varepsilon \rightarrow 0$ нижняя граница ведет себя следующим образом:

$$
r_{0}^{\varepsilon}(\Theta) \geqslant \sum_{k=1}^{\infty} s_{k}^{2}\left(1+\varepsilon^{2} \pi^{2} a_{k}^{2}\right)^{-1}=\frac{\varepsilon^{2}}{2 \beta} \ln \frac{P}{\pi^{2} \varepsilon^{2}}+\varepsilon^{2}(\gamma-\ln \pi)+o\left(\varepsilon^{2}\right) .
$$

Таким образом, между верхней и нижней границами имеется зазор, равный $\varepsilon^{2} \beta^{-1} \ln \pi$, который уменьшается с ростом гладкости $\beta$.

4.4. Нелинейное оценивание. В этом пункте мы вычислим минимаксный риск $r_{0}^{\varepsilon}(\Theta)$ в терминах задачи (2.10)-(2.12). При этом считаем, что $a_{k}^{2}=(\pi k)^{2 \beta} / P$ и $s_{k}=k^{-1 / 2}$.

Пусть, как и ранее, $N=\max \left\{k:\left|a_{k}\right|<1 / \varepsilon\right\}$ и

$$
R_{\delta}=\inf _{\widehat{L}_{\delta}} \sup _{f \in \mathscr{F}} \mathbf{E}_{\theta}\left[L_{\delta}(f)-\widehat{L}_{\delta}\right]^{2}+\ln \delta
$$

- нормированный минимаксный риск.

Лемма 6. При $\varepsilon \rightarrow 0$ справедливо неравенство

$$
r_{\varepsilon}^{2}(\Theta) \geqslant \frac{\varepsilon^{2}}{2 \beta} \ln \frac{P}{\varepsilon^{2}}+\varepsilon^{2} \gamma+\varepsilon^{2} \lim _{\delta \rightarrow 0} R_{\delta}+o\left(\varepsilon^{2}\right) .
$$

Д о к а з а т е л ь с т в о. Разобьем множество индексов $\{1,2, \ldots\}$ на два подмножества: $K_{1}=\{1, \ldots, \delta N\}$ и $K_{2}=\{\delta N+1, \ldots\}$, где $\delta$ достаточно малое число. Тогда представим оцениваемый функционал в виде $L(\theta)=L_{1}(\theta)+L_{2}(\theta)$, где

$$
L_{1}(\theta)=\sum_{k \in K_{1}} s_{k} \theta_{k}, \quad L_{2}(\theta)=\sum_{k \in K_{2}} s_{k} \theta_{k} .
$$

Далее, для некоторого числа $\alpha \in(0,1)$ определим два множества

$$
\begin{aligned}
& \Theta_{1}=\left\{\theta_{k}, k \in K_{1}: \sum_{k \in K_{1}} a_{k}^{2} \theta_{k}^{2} \leqslant \alpha\right\}, \\
& \Theta_{2}=\left\{\theta_{k}, k \in K_{2}: \sum_{k \in K_{2}} a_{k}^{2} \theta_{k}^{2} \leqslant 1-\alpha\right\} .
\end{aligned}
$$

Обозначим для краткости $\mathbf{X}_{1}=\left(X_{1}, \ldots, X_{\delta N}\right)^{T}$ и $\mathbf{X}_{2}=\left(X_{\delta N+1}, \ldots\right)^{T}$. Пусть $\pi_{1}(\theta), \theta \in \Theta_{1}$, и $\pi_{2}(\theta), \theta \in \Theta_{2}$, - априорные плотности распределения на множествах $\Theta_{1}$ и $\Theta_{2}$, а $p_{1}\left(\mathbf{X}_{1} \mid \theta\right), \theta \in \Theta_{1}$, и $p_{2}\left(\mathbf{X}_{2} \mid \theta\right), \theta \in \Theta_{2}$, плотности распределения векторов $\mathbf{X}_{1}, \mathbf{X}_{2}$.

Тогда

$$
\inf _{\widehat{L}} \sup _{\theta \in \Theta} \mathbf{E}_{\theta}\left[L_{1}(\theta)+L_{2}(\theta)-\widehat{L}\right]^{2} \geqslant \inf _{\widehat{L}} \mathbf{E} \mathbf{E}_{\theta_{1}, \theta_{2}}\left[L_{1}\left(\theta_{1}\right)+L_{2}\left(\theta_{2}\right)-\widehat{L}\right]^{2}
$$


здесь $\mathbf{E}$ - усреднение по мере, имеющей плотность $\pi_{1}\left(\theta_{1}\right) \pi_{2}\left(\theta_{2}\right)$. Заметим, что нижняя грань правой части достигается в том случае, если оценка $\widehat{L}$ байесовская, а именно

$$
\widehat{L}(X)=\frac{\int_{\Theta_{1}} \int_{\Theta_{2}}\left[L_{1}\left(\theta_{1}\right)+L_{2}\left(\theta_{2}\right)\right] p_{1}\left(\mathbf{X}_{1} \mid \theta_{1}\right) p_{2}\left(\mathbf{X}_{2} \mid \theta_{2}\right) \pi_{1}\left(\theta_{1}\right) \pi_{2}\left(\theta_{2}\right) d \theta_{1} d \theta_{2}}{\int_{\Theta_{1}} \int_{\Theta_{2}} p_{1}\left(\mathbf{X}_{1} \mid \theta_{1}\right) p_{2}\left(\mathbf{X}_{2} \mid \theta_{2}\right) \pi_{1}\left(\theta_{1}\right) \pi_{2}\left(\theta_{2}\right) d \theta_{1} d \theta_{2}}
$$

Отсюда ясно, что $\widehat{L}(X)=\widehat{L}_{1}\left(\mathbf{X}_{1}\right)+\widehat{L}_{2}\left(\mathbf{X}_{2}\right)$, где $\widehat{L}_{1}(\cdot)$ и $\widehat{L}_{2}(\cdot)$ - байесовские оценки векторов $\theta_{1}$ и $\theta_{2}$ соответственно. Так как эти оценки являются несмещенными, находим

$$
\begin{aligned}
& \underset{\widehat{L}}{\inf } \mathbf{E} \mathbf{E}_{\theta_{1}, \theta_{2}}\left[L_{1}\left(\mathbf{X}_{1}\right)-L_{2}\left(\mathbf{X}_{2}\right)-\widehat{L}\right]^{2} \\
& \quad=\mathbf{E} \mathbf{E}_{\theta_{1}}\left[L_{1}\left(\theta_{1}\right)-\widehat{L}_{1}\left(\mathbf{X}_{1}\right)\right]^{2}+\mathbf{E} \mathbf{E}_{\theta_{2}}\left[L_{2}\left(\theta_{2}\right)-\widehat{L}_{2}\left(\mathbf{X}_{2}\right)\right]^{2}
\end{aligned}
$$

Вспомним, что плотности $\pi_{1}$ и $\pi_{2}$ были выбраны произвольно, поэтому

$$
\sup _{\pi_{i}} \mathbf{E} \mathbf{E}_{\theta_{i}}\left[L_{i}\left(\theta_{i}\right)-\widehat{L}_{i}\left(\mathbf{X}_{i}\right)\right]^{2}=\sup _{\theta_{i} \in \Theta_{i}} \mathbf{E}_{\theta_{i}}\left[L_{i}\left(\theta_{i}\right)-\widehat{L}_{i}(\mathbf{X})\right]^{2}, \quad i=1,2 .
$$

Таким образом, из (4.14) и (4.15) получаем неравенство

$$
r_{0}^{\varepsilon}(\Theta) \geqslant r_{0}^{\varepsilon}\left(\Theta_{1}\right)+r_{0}^{\varepsilon}\left(\Theta_{2}\right)
$$

Поэтому наша задача распадается на две независимые задачи оценки функционалов $L_{1}(\theta), \theta \in \Theta_{1}$, и $L_{2}(\theta), \theta \in \Theta_{2}$.

Чтобы оценить снизу $r_{0}^{\varepsilon}\left(\Theta_{1}\right)$, поступим так же, как при доказательстве леммы 5. Положим $\theta_{k}=b_{k} \zeta$ при $k \in K_{1}$, где $\zeta-$ некоторая случайная величина. Условие $\theta \in \Theta_{1}$ влечет следующее ограничение на $\zeta: \zeta^{2} \sum_{k=1}^{\delta N} a_{k}^{2} b_{k}^{2} \leqslant \alpha$. Поэтому нетрудно показать (см. доказательство леммы 5), что

$$
r_{0}^{\varepsilon}\left(\Theta_{1}\right) \geqslant \varepsilon^{2} \sum_{k=1}^{\delta N} k^{-1}\left(1+\frac{\varepsilon^{2} \pi^{2} a_{k}^{2}}{\alpha}\right)^{-1}
$$

Заметим, далее, что

$$
\begin{aligned}
& \sum_{k=1}^{\delta N} k^{-1}\left(1+\frac{\varepsilon^{2} \pi^{2} a_{k}^{2}}{\alpha}\right)^{-1} \geqslant \sum_{k=1}^{\delta N} k^{-1}\left(1-\frac{\pi^{2} a_{k}^{2} \varepsilon^{2}}{\alpha}\right) \\
& \quad=\ln \delta N+\gamma+o(1)-\frac{\varepsilon^{2} \pi^{2}}{\alpha} \sum_{k=1}^{\delta N} \frac{a_{k}^{2}}{k}=\ln \delta N+\gamma+o(1)-\alpha^{-1} O\left(\delta^{2 \beta}\right) .
\end{aligned}
$$

Выбрав $\delta^{2 \beta} / \alpha=o(1), \varepsilon \rightarrow 0$, отсюда и из (4.17) получаем следующее неравенство:

$$
r_{0}^{\varepsilon}\left(\Theta_{1}\right) \geqslant \varepsilon^{2}[\ln N+\ln \delta+\gamma+o(1)]
$$


Рассмотрим теперь второе слагаемое в правой части (4.16). Заметим, что вместо оценивания функционала $L_{2}(\theta)$ мы можем оценивать функционал $L_{\delta}^{A}(\theta)=\sum_{k=\delta N}^{A N} \theta_{k} / \sqrt{k}$, так как

$$
r_{0}^{\varepsilon}\left(\Theta_{2}\right) \geqslant \inf _{\widehat{L}} \sup _{\theta \in \Theta_{2}} \mathbf{E}_{\theta}^{\varepsilon}\left[L_{\delta}^{A}(\theta)-\widehat{L}\right]^{2} .
$$

Рассмотрим новую функцию потерь

$$
w_{B}(x)= \begin{cases}x^{2}, & |x|<B \\ B^{2}, & |x| \geqslant B\end{cases}
$$

где $B$ - некоторое положительное число. Так как $x^{2} \geqslant w_{B}(x)$, то из (4.19) находим

$$
r_{0}^{\varepsilon}\left(\Theta_{2}\right) \geqslant \varepsilon^{2} \inf _{\widehat{L}} \sup _{\theta \in \Theta_{2}} \mathbf{E}_{\theta}^{\varepsilon} w_{B}\left[\varepsilon^{-1}\left(L_{\delta}^{A}(\theta)-\widehat{L}\right)\right] .
$$

Пусть $\mathscr{F}_{\alpha}(A, Q)$ - множество всех функций $f$ таких, что $f(x)=0$ при $x \notin[0, A], \sup _{x} f^{\prime}(x) \leqslant Q$ и

$$
\int_{0}^{A} x^{2 \beta} f(x) d x \leqslant 1-2 \alpha .
$$

Положим

$$
\theta_{k}=\frac{P^{1 / 2}}{N^{\beta+1 / 2} \pi^{\beta}} f\left(\frac{k}{N}\right)
$$

Поскольку производная $f^{\prime}(t)$ ограничена на отрезке $[0, A]$, то

$$
\sum_{\delta N}^{A N} a_{k}^{2} \theta_{k}^{2} \leqslant \frac{1}{N} \sum_{k=1}^{A N}\left(\frac{k}{N}\right)^{2 \beta} f^{2}\left(\frac{k}{N}\right)=\int_{0}^{A} t^{2 \beta} f^{2}(t) d t+O\left(\frac{Q}{N}\right) .
$$

Таким образом, $\theta \in \Theta_{2}$. По тем же соображениям оцениваемый функционал аппроксимируется следующим образом:

$$
L_{\delta}^{A}(\theta)=\frac{\varepsilon}{N} \sum_{k=\delta N}^{A N} \frac{f(k / N)}{\sqrt{k / N}}=\varepsilon \int_{\delta}^{A} \frac{f(t)}{\sqrt{t}} d t+o(\varepsilon) .
$$

Далее, заметим, что наблюдения, переписанные в терминах $f(\cdot)$, имеют вид

$$
X_{k}=\frac{P^{1 / 2}}{N^{\beta+1 / 2} \pi^{\beta}} f\left(\frac{k}{N}\right)+\varepsilon \xi_{k} .
$$

Отсюда, замечая, что $\sqrt{P} /\left(N^{\beta} \pi^{\beta}\right)=(1+o(1)) \varepsilon$, непосредственно приходим к следующим эквивалентным наблюдениям:

$$
Y_{k}=f\left(\frac{k}{N}\right)+(1+o(1)) \sqrt{N} \xi_{k} .
$$

Перейдем от этих наблюдений к эквивалентным наблюдениям с непрерывным временем. Обозначим через $\bar{f}(t)$ ступенчатую функцию такую, 
что $\bar{f}(t)=f(k / N)$ при $|t-k / N| \leqslant 1 /(2 N)$. Тогда легко видеть, что наблюдения (4.24) эквивалентны следующим:

$$
d \bar{Y}(t)=\bar{f}(t) d t+d w(t), \quad t \in[0, A]
$$

где $w(t)$ - стандартный винеровский процесс. Заметим, что $\|\bar{f}-f\| \rightarrow 0$ при $\varepsilon \rightarrow 0$. Это означает, что статистическая задача оценивания линейного функционала

$$
L_{\delta}^{A}(f)=\int_{\delta}^{A} \frac{f(t)}{\sqrt{t}} d t
$$

по наблюдениям (4.25) асимптотически эквивалентна (см., например, [1]) задаче оценивания этого же функционала по наблюдениям

$$
d Y(t)=f(t) d t+d w(t), \quad t \in[0, A] .
$$

В свою очередь это значит, что

$$
\begin{aligned}
& \quad \inf _{\widehat{L}} \sup _{f \in \mathscr{F}_{\alpha}(A, Q)} \mathbf{E}_{f} w_{B}\left\{L_{\delta}^{A}(f)-\widehat{L}(\bar{Y})\right\} \\
& \quad \geqslant \inf _{\widehat{L}} \sup _{f \in \mathscr{F}_{\alpha}(A, Q)} \mathbf{E}_{f} w_{B}\left\{L_{\delta}^{A}(f)-\widehat{L}(Y)\right\}+o(1) .
\end{aligned}
$$

Отсюда, из (4.23) и (4.20) вытекает, что

$$
\lim _{\varepsilon \rightarrow 0} \varepsilon^{-2} r_{0}^{\varepsilon}\left(\Theta_{2}\right) \geqslant \inf _{\widehat{L}} \sup _{f \in \mathscr{F}_{\alpha}(A, Q)} \mathbf{E}_{f} w_{B}\left[L_{\delta}^{A}(f)-\widehat{L}\right] .
$$

Предельный переход при $B \rightarrow \infty, Q \rightarrow \infty$ и $\alpha \rightarrow 0$ (см. также (4.16) и (4.18)) завершает доказательство леммы.

Теперь мы покажем, что полученная граница снизу не может быть улучшена.

Лемма 7. При $\varepsilon \rightarrow 0$ выполнено неравенство

$$
r_{0}^{\varepsilon}(\Theta) \leqslant \varepsilon^{2} \ln N+\varepsilon^{2} \gamma+\varepsilon^{2} \lim _{\delta \rightarrow 0} R_{\delta}+o\left(\varepsilon^{2}\right) .
$$

Д о к а з а т е л с с т в о. Опять разобьем наблюдения на две части, как при доказательстве нижней границы. При этом оцениваемый функционал $L(\theta)$ распадается на сумму двух функционалов $L_{1}(\theta)$ и $L_{2}(\theta)$. В качестве оценки функционала $L_{1}(\theta)=\sum_{k=1}^{\delta N} \theta_{k} / \sqrt{k}$ возьмем проекционную оценку $\widehat{L}_{1}(X)=\sum_{k=1}^{\delta N} X_{k} / \sqrt{k}$. Легко видеть, что тогда риск оценивается сверху следующим образом:

$$
\begin{aligned}
r_{0}^{\varepsilon}(\Theta) & \leqslant \inf _{\widehat{L}_{2}} \sup _{\theta \in \Theta} \mathbf{E}_{\theta}^{\varepsilon}\left[L_{1}(\theta)+L_{2}(\theta)-\widehat{L}_{1}(X)-\widehat{L}_{2}(X)\right]^{2} \\
& =\varepsilon^{2} \sum_{k=1}^{\delta N} k^{-1}+\inf _{\widehat{L}_{2}} \sup _{\theta \in \Theta} \mathbf{E}_{\theta}^{\varepsilon}\left[L_{2}(\theta)-\widehat{L}_{2}(X)\right]^{2}
\end{aligned}
$$


Отсюда ясно, что нужно оценить сверху последнее слагаемое. Для этого сведем задачу с непрерывным временем (2.10)-(2.12) к исходной дискретной задаче. Точнее, мы докажем, что

$$
\begin{aligned}
& \inf _{\widehat{L}_{2}} \sup _{\theta \in \Theta} \mathbf{E}_{\theta}^{\varepsilon}\left[L_{2}(\theta)-\widehat{L}_{2}(X)\right]^{2}+\varepsilon^{2} \ln \delta \\
& \quad \leqslant \varepsilon^{2} \inf _{\widehat{L}_{\delta}} \sup _{f \in \mathscr{F}} \mathbf{E}_{f}\left[\widehat{L}_{\delta}(X)-L_{\delta}(f)\right]^{2}+\varepsilon^{2} \ln \delta+o\left(\varepsilon^{2}\right)
\end{aligned}
$$

здесь функционал $L_{\delta}(f)$ определен в $(2.10)$. Пусть $\overline{\mathscr{F}}-$ множество всех ступенчатых функций из $\mathscr{F}$ вида

$$
\bar{f}(x)=\sum_{k=\delta N}^{\infty} f\left(\frac{k}{N}\right) 1\left\{\frac{k}{N} \leqslant x<\frac{k+1}{N}\right\} .
$$

Тогда очевидно, что для любой оценки $\widehat{L}_{\delta}(X)$ справедливо неравенство

$$
\sup _{f \in \mathscr{F}} \mathbf{E}_{f}\left[L_{\delta}(f)-\widehat{L}_{\delta}(X)\right]^{2} \geqslant \sup _{f \in \mathscr{F}} \mathbf{E}_{f}\left[L_{\delta}(f)-\widehat{L}_{\delta}(X)\right]^{2}
$$

В то же время наблюдения (2.11) эквивалентны следующим:

$$
X_{k}^{\varepsilon}=f\left(\frac{k}{N}\right)+\sqrt{N} \xi_{k} .
$$

Пусть $\theta_{k}$ определены в (4.22). Тогда наблюдения (4.30) эквивалентны

$$
Z_{k}^{\varepsilon}=\theta_{k}+(1+o(1)) \varepsilon \xi_{k} .
$$

Оцениваемый функционал в терминах $\theta_{k}$ принимает вид

$$
\begin{aligned}
L_{\delta}(\bar{f}) & =\int_{\delta}^{\infty} \frac{\bar{f}(t)}{\sqrt{t}} d t=\frac{\pi^{\beta} N^{\beta+1 / 2}}{\sqrt{P}} \sum_{k=\delta N}^{\infty} \theta_{k} \int_{k / N}^{(k+1) / N} \frac{d t}{\sqrt{t}} \\
& =\frac{1+o(1)}{\varepsilon} \sum_{k=\delta N}^{\infty} \frac{\theta_{k}}{\sqrt{k}}-\frac{1+o(1)}{\varepsilon} \sum_{k=\delta N}^{\infty} \theta_{k} \frac{1}{\sqrt{k}(\sqrt{k+1}+\sqrt{k})^{2}} .
\end{aligned}
$$

Ограничения на $\bar{f}$ пересчитываются в ограничения на $\theta_{k}$ следующим образом:

$$
\begin{aligned}
1 & \geqslant \int_{0}^{\infty} t^{2 \beta} \bar{f}^{2}(t) d t=\frac{\pi^{2 \beta}}{P(2 \beta+1)} \sum_{k=\delta N}^{\infty} \theta_{k}^{2}\left[(k+1)^{2 \beta+1}-k^{2 \beta+1}\right] \\
& \geqslant \frac{\pi^{2 \beta}}{P} \sum_{k=\delta N}^{\infty} \theta_{k} k^{2 \beta}
\end{aligned}
$$

T.e.

$$
\sum_{k=\delta N}^{\infty} a_{k}^{2} \theta_{k}^{2} \leqslant 1
$$


Поэтому, отсюда и из (4.32), применяя неравенство Коши-Буняковского, получаем

$$
\left[\varepsilon L_{\delta}(\bar{f})-\sum_{k=\delta N}^{\infty} \frac{\theta_{k}}{\sqrt{k}}\right]^{2} \leqslant O\left((\delta N)^{-2 \beta-2}\right)+o\left((\delta N)^{-2 \beta}\right) .
$$

Таким образом, из этого неравенства, воспользовавшись (4.33), (4.31) и (4.29), получаем (4.28). Утверждение леммы теперь непосредственно вытекает из (4.27).

Д ок а з а тель с т в о т е ре мы 3 следует непосредственно из лемм 6 и 7.

\section{СПИСОК ЛИТЕРАТУРЫ}

1. Brown L. D., Low M. G. Asymptotic equivalence of nonparametric regression and white noise. - Ann. Statist., 1996, v. 24, p. 2384-2398.

2. Van Es B., Hoogendoorn A. W. Kernel estimation in Wicksell's corpuscle problem. Biometrika, 1990, v. 77, №1, p. 139-145.

3. Golubev G., Levit B. Asymptotically efficient estimation in the Wicksell problem. Ann. Statist., 1998, v. 26, p. 2407-2419.

4. Groeneboom P., Jongbloed G. Isotonic estimation and rates of convergence in Wicksell's problem. - Ann. Statist., 1995, v. 23, p. 1518-1542.

5. Hall P., Smith R. L. The kernel method for unfolding sphere size distributions. - J. Comput. Phys., 1988, v. 74, p. 409-421.

6. Hoogendoorn A.W. Estimating the weight undersize distribution for the Wicksell problem. - Statist. Neerlandica, 1992, v. 4, p. 259-282.

7. Nussbaum $M$. Asymptotic equivalence of density estimation and Gaussian white noise. - Ann. Statist., 1996, v. 24, p. 2399-2430.

8. Taylor C. C. A new method for unfolding sphere size distributions. - J. Microscopy, 1983 , v. 132 , p. $57-66$.

9. Watson D.S. Estimating functionals of particle size distributions. - Biometrika, 1971 , v. 58 , p. $483-490$.

10. Wicksell $S$. D. The corpuscle problem. A mathematical study of a biometric problem. - Biometrika, 1925 , v. 17, p. 84-99.

11. Ван Трис Г. Л. Теория обнаружения, оценок и модуляции. Т. 1. М.: Сов. радио, 1975.

12. Зигмунд А. Тригонометрические ряды. Т. 2. М.: Мир, 1965, 537 с.

13. Ибрагимов И. А., Хасьминский Р. З. Асимптотическая теория оценивания. М.: Наука, 1979, $528 \mathrm{c}$.

14. Пинскер M. С. Оптимальная фильтрация квацратично-интегрируемых сигналов на фоне гауссовского белого шума. - Проблемы передачи информации, 1980, т. 16 , в. 2. с. $52-68$. 